\title{
Changes in ST, QT and RR ECG intervals during acute stress in firefighters: a pilot study
}

\author{
Joana S. Paiva ${ }^{1}$, Susana Rodrigues ${ }^{1}$ and João Paulo Silva Cunha ${ }^{1}$
}

\begin{abstract}
Firefighting is a stressful occupation. The monitoring of psychophysiological measures in those professionals can be a way to prevent and early detect cardiac diseases and other stress-related problems. The current study aimed to assess morphological changes in the ECG signal induced by acute stress. A laboratory protocol was conducted among 6 firefighters, including a laboratory stress-inducer task - the Trier Social Stress Task (TSST) - and a 2-choice reaction time task (CRTT) that was performed before (CRTT1) and after (CRTT2) the stress condition. ECG signals were continuously acquired using the VitalJacket ${ }^{\circledR}$, a wearable $t$-shirt that acts as a medical certified ECG monitor. Results showed that ECG morphological features such as QT and ST intervals are able to differentiate stressful from non stressful events in first responders. Group mean Visual Analogue Scale (VAS) for stress assessment significantly increased after the stress task (TSST), relatively to the end of CRTT2 (after TSST: $4.67 \pm 1.63$; after CRTT2: $3.17 \pm 0.75$ ), a change that was accompanied by a significant increase in group mean QT and ST segments corrected for heart rate during TSST. These encouraging results will be followed by larger studies in order to explore those measures and its physiological impact under realistic environments in a higher scalability.
\end{abstract}

\section{INTRODUCTION}

Firefighting is a stressful occupation that, consequently, lead to a higher risk of coronary diseases, which is responsible for a large percentage of deaths among firefighters (FFs) [1]. Epidemiologic studies reported that, for the population in general, stress and cardiac morbidity and mortality are tightly correlated [2]. It is already known that acute stress induces physiological responses by our cardiovascular and neuroendocrine systems [3]. Currently, frequency-derived ECG measures, such as the heart rate variability (HRV), are being used for monitoring the effects of stress in the cardiovascular system [4]. However, there are recent evidences that stress can also induce changes in the morphology of the ECG waveforms [3], [5] that can also be measured. The monitorization of those changes reveals to be important, particularly in high risk professionals, given that several cardiovascular diseases such as arrhythmias, ventricular hypertrophy, electrolyte imbalance, are related with abnormal ECG morphological alternans [5].

The most frequently morphological measures used to identify ECG abnormal patterns are the time intervals between fiducials points [5], [6]. The fiducial points most commonly

\footnotetext{
*This work has been financed by National Funds through the FCT (Portuguese Foundation for Science and Technology) within the project VR2Market CMUP-ERI/FIA/0031/2013 and PhD Grant PD/BI/114377/2016. We would like to thank the Chief Command of the fire department and the firefighters for accepted to participate in this study.

${ }^{1}$ INESC TEC and Faculty of Engineering, University of Porto.
}

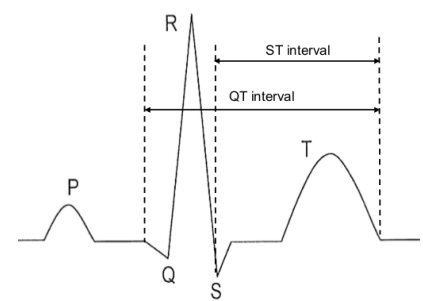

Fig. 1. Fiducial Points most commonly analyzed; $S T$ and $Q T$ intervals.

used are the Q, R, S and T points - see figure 1. According to the literature, the time intervals between fiducials that are significantly affected by stress are $R R, S T$ and $Q T$ intervals [5], [6]. Recently, it was found that also $R R, P P$ intervals and $S T$ segments can differentiate stressful tasks in FFs [7]. Particularly, $Q T$ interval traduces the interval between the onset of electrical activation and its recovery, reflecting the balance of the sympathetic and parasympathetic nervous systems [3]. ST interval reflects ventricular activation, being also influenced by external stimuli such as exercise, despite no other study, as far as we know, has investigated directly the influence of stressful events in the $S T$ interval in a laboratory environment. However, both ECG intervals are strongly dependent on the heart rate (HR) [3], [5]. Consequently, a normalization method must be applied to $S T$ and $Q T$ intervals when these two measures are compared between different conditions. The method most commonly used for correcting HR-dependent features is the Bazett Formula [8], [9].

Considering that the few studies developed so far reported conflicting results [3], [5], [10] and no other study, unless the one conducted by our affiliated lab about 3 years ago [7], studied stress-induced ECG morphological changes in FFs during stressful tasks, we applied a laboratory protocol with FFs in order to understand how acute stress can affect ECG morphology. In this study, we hypothesized that the morphological features $R R$ interval and $S T$ and $Q T$ intervals corrected for HR (named here as $S T_{c}$ and $Q T_{c}$, respectively) could differentiate stressful from non stressful events in FFs.

\section{Methods}

\section{A. Description of the laboratory protocol}

Six FFs (1 female, 5 male; age: $35 \pm 13$ ) from a Portuguese Firefighter unit volunteered to participate in this study, after signed an informed consent. The exclusion criteria for the study were participants having a history 


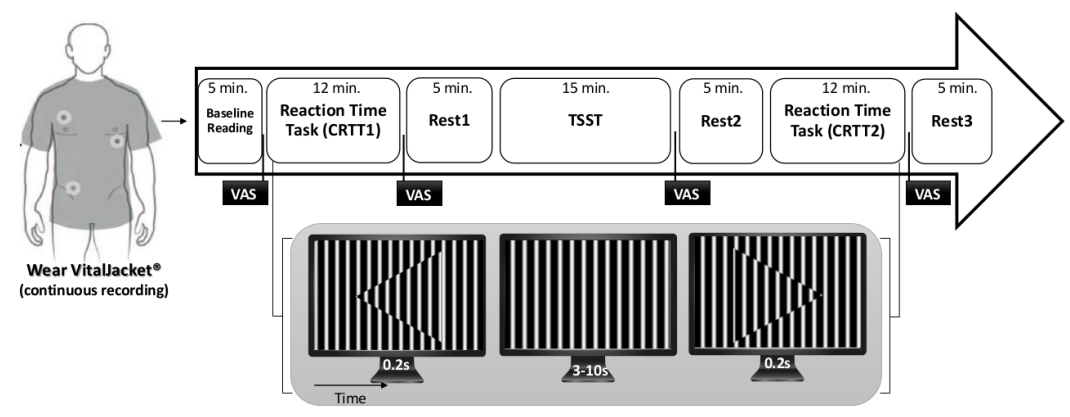

Fig. 2. Diagram of the protocol. VAS - Visual Analogue Scales. TSST - Trier Social Stress Test.

of cardiovascular disease and/or taking prescription drugs known to affect cardiovascular function.

ECG signals were continuously acquired using the VitalJacket ${ }^{\circledR}$ [11] (VJ) at $500 \mathrm{~Hz}$ from a single lead, totaling approximately 22260 minutes of ECG acquired among the 6 FFs (including all the stages of the protocol; figure 2). Note that only the heartbeats ( $\mathrm{HBs}$ ) of specific protocol stages were analyzed, as explained in subsection II-C. The VJ is a wearable bio-monitoring platform (in form of a t-shirt) that provides real time ECG without interfering with the user daily activities. It is also composed by a three axis Accelerometer (ACC) system, that is able to monitor the level of user activity and posture. The laboratory protocol was composed of 3 main tasks - see figure 2. Subjects were comfortably sat in a chair during this time. After a baseline reading period, a 2-choice reaction time task (CRTT1), created using custom-made MATLAB ${ }^{\circledR}$ scripts and adapted from the study of Paiva [12], was performed. In this task, FFs had to respond to a stimuli (an arrowhead pointing to the left or the right) that appeared in a PC screen, by clicking a button as fast as was possible, according with the direction of the arrowhead. Therefore, the Trier Social Stress Test (TSST) [13] - an acute stress assessment task - was applied. After subjects being exposed to the stress condition - the TSST -, they performed again the simple CRTT (CRTT2) described above. Reaction time tasks were applied in order to evaluate the impact of stress in the cognitive performance of FFs. Visual Analoge Scales (VAS) [14] were used for stress psychological self assessment after each main task. Participants were allowed to rest for 5 minutes after each one of the tasks, as explained in figure 2.

\section{B. ECG processing and extraction of $R R, Q T_{c}$ and $S T_{c}$}

Processing was done using custom-made scripts using MATLAB R2015a. At first, fiducial points Q, R, S and $\mathrm{T}$ were located on the raw signal - see figure 3 . The $\mathrm{R}$ points were located using the Pan Tompkins algorithm [15], which has been extensively used for the last 2 decades in the majority of the studies related with ECG waveform [16]. Considering that, previous studies proved that the best method for detecting ECG fiducial points is based on low order polynomial filtering [17], the raw signal was filtered using a second order Butterworth low-pass filter with a cut off frequency of $10 \mathrm{~Hz}$, in order to locate the remaining points $(\mathrm{Q}, \mathrm{S}$ and $\mathrm{T})$. All the fiducial points were located based on previously established physiological time intervals [18]. The Q points were identified by computing the signal derivative considering a time window of 0.10 seconds defined before each $\mathrm{R}$ point. The last temporal mark (and the closest one relatively to each $\mathrm{R}$ complex) at which the derivative signal crossed zero considering this time window was marked as point $\mathrm{Q}$ for each HB. A similar method was used for locating the point S. The first temporal mark at which the derivative changed from negative to positive values, in a time window of 0.05 seconds after each point $R$, was assigned as the point $\mathrm{S}$. For locating the $\mathrm{T}$ wave, it was determined the last temporal index where the derivative of the signal changed from positive to negative values, between 0.05 and 0.40 seconds after each $\mathrm{R}$ complex.

For computing QT interval, the index of the beginning of QT interval - see figures 1 and 3 - was located by determining the last point where the derivative changed from positive to negative in a time window of 0.03 seconds before each $\mathrm{Q}$ point. The index of the end of each QT interval was assigned as the index of the end of the $\mathrm{T}$ wave (the last point at which the signal derivative changed from negative to positive values within 0.15 seconds after $\mathrm{T}$ point). $S T$ interval was assigned as the interval between each fiducial point $\mathrm{S}$ and the index of the end of the corresponding $\mathrm{T}$ wave - see figure 1. $R R$ intervals were defined as the

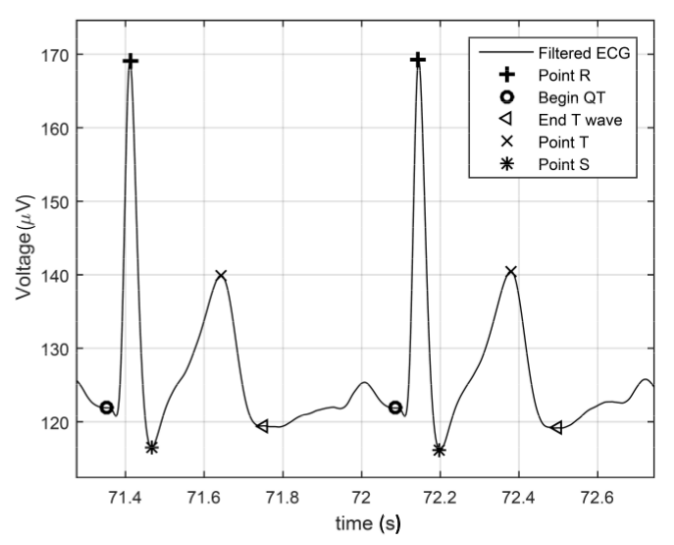

Fig. 3. Portion of ECG processed from FF2 with fiducial points $\mathrm{R}, \mathrm{S}$ and $\mathrm{T}$; and points that contributed for extracting $Q T$ and $S T$ intervals marked. 
interval between two consecutive $\mathrm{R}$ points. Time intervals $Q T, S T$ and $R R$ were calculated for each HB. In order to correct $Q T$ and $S T$ intervals for the interference of HR, the Bazett Formula [8] was applied to $Q T$ and $S T$ intervals of each HB: $Q T_{C}=\frac{Q T}{\sqrt{R R}}$ and $S T_{C}=\frac{S T}{\sqrt{R R}}$, where $Q T_{C}$ and $S T_{C}$ correspond to $Q T$ and $S T$ intervals corrected for $\mathrm{HR}$, respectively. Therefore, noisy $\mathrm{HBs}$ were removed after identifying the HBs which did not satisfy the following conditions: $Q R \leq 0.075 s$ and $0.200 s<Q T_{C}<0.360 s$ [18]. Only $Q T_{C}, S T_{C}$ and $R R$ were used in statistical analysis.

\section{Statistical Analysis}

Non parametric tests were applied, considering the reduced population sample, and also due to some of the variables analyzed failed to be normal distributed (ShapiroWilk Normality Test). Statistical evaluation was conducted using the Statistics Toolbox from MATLAB R2015a.

Stress VAS was compared between the different moments along the protocol at which participants reported their stress levels: before CRRT1 and after CRTT1; after CRTT1 and after TSST; after TSST and after CRTT2.

$Q T_{C}, S T_{C}$ and $R R$ values converted to z-score were also compared between the following stages: during CRTT1 and during rest1; during rest1 and during TSST; during TSST and during rest2; during rest2 and during CRTT2; during CRTT2 and during rest3. Note that Stress VAS was not reported during all the events, being only reported at the beginning of CRTT1 and at the end of the three principal tasks: CRTT1, TSST and CRTT2. The former 5 statistical comparisons were performed by selecting an equal number of $\mathrm{HB}$ for each condition, in order to avoid significant differences between the number of HB that contributed for the mean value of $Q T_{C}, S T_{C}$ and $R R$ considered for each subject and condition, in the group statistical analysis. For each subject, the minimum number of HB between the 6 stages of the protocol (CRTT1, rest1, TSST, rest2, CRTT2 and rest3) was randomly selected for each stage, in order to ensure that the number of $\mathrm{HB}$ was balanced for both conditions, for the same subject, in each comparison made.

The average number of $\mathrm{HB}$ across subjects considered was $236 \pm 63$. The mean rate of non-usable $\mathrm{HB}$ (which were removed for containing noise) across the 6 stages of the protocol (CRTT1, rest1, TSST, rest2, CRTT2 and rest3) and across subjects was $8.26 \pm 2.71 \%$.

Wilcoxon test (two-tailed) was used for both the stress VAS and cardiac intervals group comparisons.

\section{RESULTS AND DISCUSSION}

Stress VAS significantly increased after the stress condition (TSST) - 3.17 \pm 0.75 (post CRTT1) versus $4.67 \pm 1.63$ (post TSST) - and significantly decreased after the second cognitive task to $3.17 \pm 0.75$ (post CRTT2) - p-value $<0.05$, Wilcoxon - see figure 4. Based on this self assessment measure (VAS), we can conclude that the stress condition (TSST) has induced stress in participants.

We also found that cardiac intervals $S T_{C}$ and $Q T_{C}$ significantly changed only between TSST and the resting period rest2 - p-value $<0.05$, Wilcoxon - see figure 5. The TSST induced a significant prolongation in both $S T_{C}$ and $Q T_{C}$. Interestingly, $R R$ intervals were not significantly affected by the stress condition (p-value $>0.05$; Wilcoxon), despite group mean $R R$ was lower in TSST, in comparison with rest 2 as expected $-0.056 \pm 0.312$ versus $0.137 \pm 0.288$ (zscore values). This result suggests that the morphological changes of $S T_{C}$ and $Q T_{C}$ were not related with changes in $\mathrm{HR}$ and the normalization formula used has avoided HR interference. $S T_{C}$ and $Q T_{C}$ were not also correlated in both the TSST and rest 2 conditions $(\mathrm{p}>0.05$; Spearman test), suggesting that any changes that occurred with those intervals were not correlated. Prolonged $Q T_{C}$ interval may be linked with changes related with electrical repolarization of the heart muscle after each contraction, reflecting inhomogeneous repolarization patterns triggered by emotional stress events [3], [5]. It is known that acute stress triggers an activation of the sympathetic nervous system, which is widespread throughout the heart, due to right and left autonomic nerves being asymmetrical distributed over the ventricules. Recent evidences show that unilateral brain activity triggered by acute stress, in addition with the imbalance of the distribution of autonomic nerves relatively to the midline, causes asymmetrical sympathetic stimulation, which leads to abnormalities in ECG pattern, as the prolongation of $Q T_{C}$ interval [5], [6], [19], that is associated with a greatly increased risk of arrhythmia and sudden death [19]. Despite, as far as we know, no other study investigated the effect of acute stress in the $S T_{C}$ intervals, Baky et al. [20] found an evidence that prolonged $S T_{C}$ is associated with high stress levels in rats. $S T$ interval prolongation is currently related with cardiomyopathy and other heart diseases in humans [21]. However, more studies should be conducted with a larger population in order to confirm these results.

\section{Conclusions}

Evidences were found that, in addition to HRV-related measures, ECG morphological characteristics such as QT and ST intervals can also provide useful information for studying the physiological consequences of acute stress. Applied implications of this study are the advantages of psychophysiological monitoring of these professionals, in order to prevent cardiac diseases triggered by abnormalities in the cardiovascular system. Future studies should be conducted in a higher scale, in order to attain more reliable and solid conclusions about the impact of acute stress in the morphological characteristics of ECG in first responders.

\section{REFERENCES}

[1] P. Young, S. Partington, M. Wetherell et al., "Stressors and Coping Strategies of UK Firefighters during On-duty Incidents," Stress and Health, vol. 30, no. 5, pp. 366-376, 2014.

[2] S. Richter, J. Brugada, R. Brugada, and P. Brugada, "Electrocardiographic markers of arrhythmic events and sudden death in channelopathies," The ECG Handbook of Contemporary Challenges, p. 107, 2015.

[3] G. Andrássy, "The effect of different stressors on the QT interval and the T wave," Orvosi Hetilap, vol. 150, no. 10, pp. 447-457, 2009. 


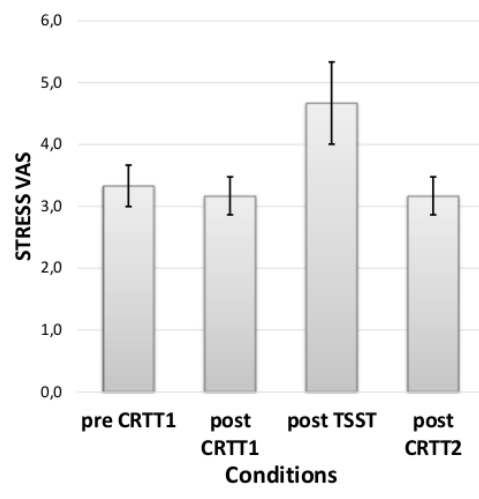

(I)

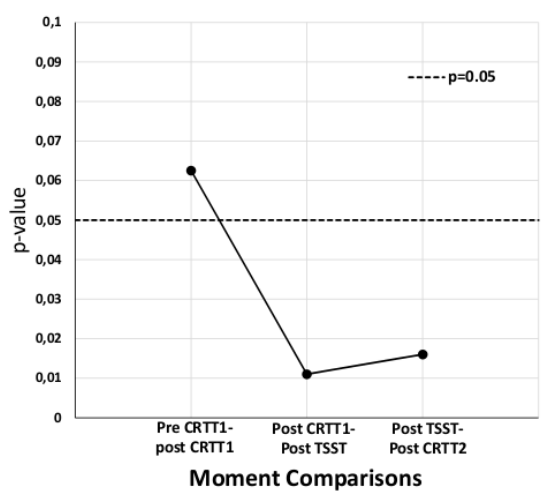

(II)

Fig. 4. Stress VAS results of the comparisons between different protocol stages. (I) Graphic illustrating VAS average across subjects (N=6) for the moments of the protocol. Error bars represent standard errors. (II) Graphic representing the evolution of p-value along those comparisons. (a) $\mathrm{p}=0.063$; (b) $\mathrm{p}=0.011 ;$ (c) $\mathrm{p}=0.016$.

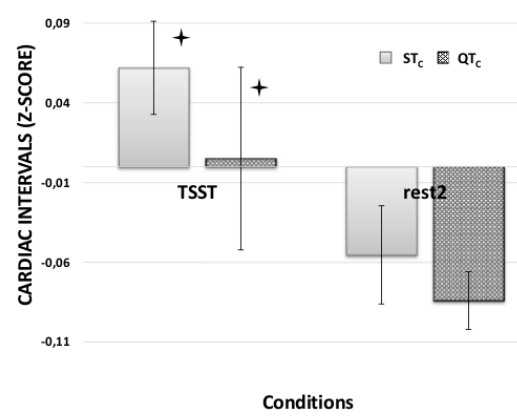

(I)

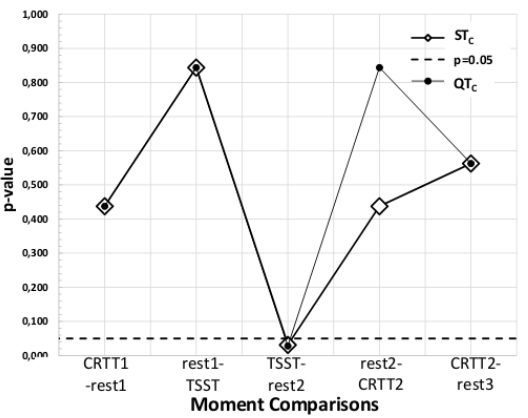

(II)

Fig. 5. Cardiac intervals with statistical significance along the protocol. (I) Graphic showing average z-score for the cardiac intervals that significantly changed between different stages of the protocol. (II) Graphic illustrating the evolution of the p-value between different moments comparisons. Considering that none of the 5 comparisons showed to be statistically significant for the $R R$ interval, $R R$ curve is not plotted here. Dashed line: statistical threshold of $\mathrm{p}$-value $=0.05$.

[4] R. Castaldo, P. Melillo, and L. Pecchia, "Acute Mental Stress Assessment via Short Term HRV Analysis in Healthy Adults: A Systematic Review," in 6th European Conference of the International Federation for Medical and Biological Engineering. Springer, 2015, pp. 1-4.

[5] A. Bhide, R. Durgaprasad, L. Kasala et al., "Electrocardiographic changes during acute mental stress," International Journal of Medical Science and Public Health (Online First), vol. 5, no. 5, 2016.

[6] M. Huerta-Franco, F. Vargas-Luna, and I. Delgadillo-Holtfort, "Effects of psychological stress test on the cardiac response of public safety workers: alternative parameters to autonomic balance," in Journal of Physics: Conference Series, vol. 582, no. 1, 2015, p. 012040.

[7] S. Bras, J. Fernandes, and J. Cunha, "ECG delineation and morphological analysis for firefighters tasks differentiation," in Proceedings of the 26th IEEE International Symposium on Computer-Based Medical Systems, 2013.

[8] H. Bazett, "An analysis of the time-relations of electrocardiograms," Heart, vol. 7, pp. 353-370, 1920.

[9] L. Zhang, K. Timothy, G. Vincent et al., "Spectrum of ST-T-wave patterns and repolarization parameters in congenital long-QT Syndrome ECG findings identify genotypes," Circulation, vol. 102, no. 23, pp. 2849-2855, 2000.

[10] C. Karmakar, M. Imam, A. Khandoker, and M. Palaniswami, "Influence of psychological stress on QT interval," in Computing in Cardiology Conference (CinC), 2014. IEEE, 2014, pp. 1009-1012.

[11] J. Cunha, B. Cunha, A. Pereira et al., "Vital-jacketß: A wearable wireless vital signs monitor for patients' mobility in cardiology and sports," in Pervasive Computing Technologies for Healthcare, 2010 4th International Conference on. IEEE, 2010, pp. 1-2.

[12] J. Paiva, "Predicting lapses in attention: a study of brain oscillations, neural synchrony and eye measures," MSc Thesis, University of
Coimbra, pp. 33-36, 2014.

[13] M. Birkett, "The Trier Social Stress Test protocol for inducing psychological stress," Journal of visualized experiments: JoVE, vol. 19, no. $56,2011$.

[14] F. Lesage, S. Berjot, and F. Deschamps, "Clinical stress assessment using a visual analogue scale," Occupational medicine, no. 62, p. 140, 2012.

[15] J. Pan and W. Tompkins, "A real-time QRS detection algorithm," Biomedical Engineering, IEEE Transactions on, vol. 32, no. 3, pp. 230-236, 1985.

[16] R. Rani, V. Chouhan, and H. Sinha, "Automated Detection of QRS Complex in ECG Signal using Wavelet Transform," International Journal of Computer Science and Network Security (IJCSNS), vol. 15, no. 1, p. 1, 2015.

[17] S. Israel, J. Irvine, A. Cheng et al., "ECG to identify individuals," Pattern recognition, vol. 38, no. 1, pp. 133-142, 2005.

[18] D. Clifford, "ECG statistics, noise, artifacts, and missing data," Advanced Methods and Tools for ECG Data Analysis, vol. 6, pp. 55-99, 2006.

[19] R. Ziegelstein, "Acute emotional stress and cardiac arrhythmias," Jama, vol. 298, no. 3, pp. 324-329, 2007.

[20] N. Baky and A. Ali, "Diazepam potentiates the protective effect of simvastatin against psychological stress-enhancement of doxorubicin cardiomyopathy," International Journal of Academic Research, vol. 1, no. 1, pp. 59-67, 2009

[21] S. Postma, J. Dambrink, A. Gosselink et al., "The influence of system delay on 30-day and on long-term mortality in patients with anterior versus non-anterior ST-segment elevation myocardial infarction: a cohort study," Open heart, vol. 2, no. 1, p. 201, 2015. 